\title{
Automating the Drug Scheduling with Different Toxicity Clearance in Cancer Chemotherapy via Evolutionary Computation
}

\author{
Yong Liang \\ Department of Computer \\ Science and Engineering \\ The Chinese University of \\ Hong Kong \\ Shatin, N.T., HK, China \\ yliang@cse.cuhk.edu.hk
}

\author{
Kwong-Sak Lueng \\ Department of Computer \\ Science and Engineering \\ The Chinese University of \\ Hong Kong \\ Shatin, N.T., HK, China \\ ksleung@cse.cuhk.edu.hk
}

\author{
Tony Shu Kam Mok \\ Department of Clinical \\ Oncology \\ The Chinese University of \\ Hong Kong \\ Shatin, N.T., HK, China \\ tony@clo.cuhk.edu.hk
}

\begin{abstract}
The toxicity of an anticancer drug is cleared from the body by different processes, including saturable metabolic and nonsaturable renal-excretion pathways. According to the principles of toxicokinetics, we propose a new anticancer drug scheduling model with different toxic elimination processes in this paper. We also present a sophisticated automating drug scheduling approach based on evolutionary computation and computer modeling. To explore multiple efficient drug scheduling policies, we use a multimodal optimization algorithm - adaptive elitist-population based genetic algorithm (AEGA) to solve the new model, and discuss the situation of multiple optimal solutions under different parameter settings. The simulation results obtained by the new model match well with the clinical treatment experience, and can provide much more drug scheduling policies for a doctor to choose depending on the particular conditions of the patients.
\end{abstract}

\section{Categories and Subject Descriptors}

I.6 [Simulation and Modeling]: Model Developmentmodeling methodologies.

\section{General Terms}

Algorithms

\section{Keywords}

Drug scheduling model, multimodal optimization algorithm

\section{INTRODUCTION}

An important target for cancer chemotherapy is to maximally kill tumor cells for a fixed treatment period. So a

Permission to make digital or hard copies of all or part of this work for personal or classroom use is granted without fee provided that copies are not made or distributed for profit or commercial advantage and that copies bear this notice and the full citation on the first page. To copy otherwise, to republish, to post on servers or to redistribute to lists, requires prior specific permission and/or a fee.

GECCO'06, July 8-12, 2006, Seattle, Washington, USA.

Copyright 2006 ACM 1-59593-186-4/06/0007 ...\$5.00. choice of anticancer drug scheduling is essential in cancer chemotherapy. The effects of anticancer drugs, as proposed by Martin [8], are given by the following differential equations:

$$
\begin{aligned}
\frac{d x_{1}}{d t} & =-\lambda x_{1}+k\left(x_{2}-\beta\right) H\left(x_{2}-\beta\right) \\
\frac{d x_{2}}{d t} & =u-\gamma x_{2} \\
\frac{d x_{3}}{d t} & =x_{2}
\end{aligned}
$$

with the initial state $x^{T}(0)=[\ln (100), 0,0]$, the parameters $\lambda=9.9 \times 10^{-4}$ day, $k=8.4 \times 10^{-3}$ day $^{-1} \times D^{-1}, \beta=10$, $\gamma=0.27$ day, $\eta=0.4 d^{-1} y^{-1}$, and:

$$
H\left(x_{2}-\beta\right)=\left\{\begin{array}{lll}
1, & \text { if } & x_{2} \geq \beta \\
0, & \text { if } & x_{2} \leq \beta
\end{array}\right.
$$

where $x_{1}$ is a transformed variable that is inversely related to the mass of the tumor. The initial tumor cell population is set at $10^{10}$ cells [8]. The variable $x_{2}$ is the drug concentration in the body in drug units $(D)$ and $x_{3}$ is the cumulative drug toxicity in the body. Equation (1) describes the net change in the tumor cell population per unit time. The first term on the right-hand side of Equation (1) describes the increase in cells due to cell proliferation and the second term describes the decrease in cells due to the drug. The parameter $\lambda$ is a positive constant related to the growth speed of the cancer cells, and $k$ is the proportion of tumor cells killed per unit time per unit drug concentration which is assumed to be a positive constant. The implication of the function described in Equation (4) is that there is a threshold of the drug concentration level, $\beta$ below which the number of the killed tumor cells is smaller than the number of the reproduced tumor cells, and the drug is not efficient. Equation (2) describes the net increase in the drug concentration at the cancer site. The variable $u$ is the rate of the delivery of the drug, and the half-life of the drug is $\ln (2) / \gamma$, where $\gamma$ is the biochemical character parameter of the drug. Equation (3) relates the cumulative drug toxicity to the drug concentration, e.g., the cumulative effect is the integral of the drug concentration over the period of exposure. 
The performance index [8] to be maximized is:

$$
I=x_{1}\left(t_{f}\right)
$$

where the final time $t_{f}=84$ days. The control optimization is performed subject to constraints on the drug delivery:

$$
u \geq 0
$$

and on the state variables:

$$
\begin{aligned}
& x_{2} \leq 50 \\
& x_{3} \leq 2.1 \times 10^{3}
\end{aligned}
$$

In this paper, we discuss about the combination of the different drug toxic elimination processes for whole-body physiological toxicokinetic model. The main inherent idea is the fundamental component of toxicokinetic modeling is that of organ clearance. In [5] [6], we have proposed three toxicokinetic models, which may be best illustrated by considering a single organ that is perfused by blood and is capable of eliminating the drug. These models with the different elimination components provide the basic units of the physiological toxicokinetic models.

The rest of this paper is organized as follows. Section 2 discusses related work on drug scheduling models for cancer chemotherapy. Section 3 presents the new drug scheduling model based on toxicokinetics. Section 4 describes the automation of the drug scheduling for cancer chemotherapy through a multimodal optimization algorithm - adaptive elitist-population based genetic algorithm (AEGA). Section 5 provides the simulation results under the new drug scheduling model. The parameter analysis and discussion are given in Section 6. The paper conclusion and future work are summarized in Section 7.

\section{RELATED WORK}

Many researchers have dealt with the Martin's drug scheduling model, however, problematic simulation results were obtained [1] [2] [7] [8] [10] [13]. In the 84 days treatment, a best-known control policy is that it first gives drug on the $42 t h$ day. It obviously does not correspond with the clinical experience. In the clinical treatment, giving the multi-dose drug at the first day can get the best efficiency of the cancer treatment: to kill maximal tumor cells with minimal drug toxicity.

To solve the above unreasonable problem and accurately describe the time course of the cumulative drug toxicity $x_{3}$ in the body, in [6], we have modified the third equation in Martin's model as follows:

$$
\frac{d x_{3}}{d t}=x_{2}-\eta_{E} \times x_{3} .
$$

Equation (9) describes the net change of the cumulative drug toxicity $x_{3}$ per unit time. In the right-hand side of this equation, the first term $x_{2}$ describes the increase of the cumulative drug toxicity $x_{3}$ due to the drug concentration $x_{2}$, and the second term $\eta_{E} \times x_{3}$ can be called the exponential drug toxicity clearance function, which describes the decrease in the drug toxicity due to the clearance in the body. Thus, a drug toxic elimination process is described as the first-order elimination and the proportionality constant $\eta_{E}$ is known as the drug toxic elimination rate constant. The

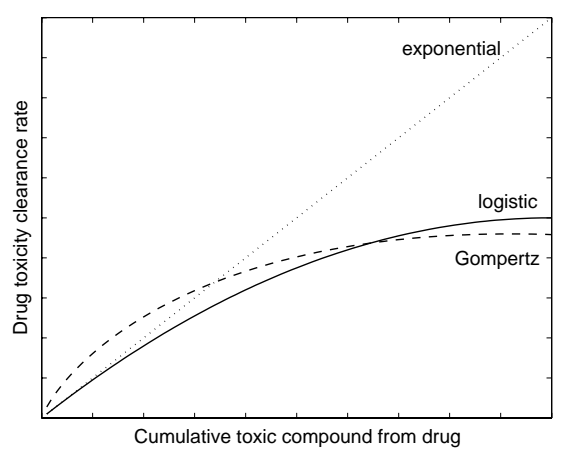

Figure 1: Three monotonically increasing functions - exponential, logistic and Gompertz - are used here to simulate different drug toxic elimination processes.

first-order elimination is by far the most common kinetic model to describe nonsaturable toxic elimination pathway, e.g. filtration in renal excretion.

However, the metabolism of many anticancer drugs is enzymatic reactions in organs and tissues. Drug-metabolizing enzymes are saturable. This means that the different numbers of substrate (the cumulative toxic compound) affect the rate of the enzymatic reaction (the speed of the toxic elimination). When the amount of the cumulative toxic compound is small, the speed of the toxicity clearance is proportional to the amount of the cumulative toxic compound. However, as the cumulative toxic compound is increased, the speed of the toxicity clearance falls off and is no longer proportional to the amount of the cumulative toxic compound; in this zone, the toxic elimination is of mixed-order. On further increase in the cumulative toxic compound, the rate becomes almost constant and independent of the cumulative toxic compound. In this range of the cumulative toxic compound, the toxic elimination is zero-order with respect to the amount of the cumulative toxic compound [3].

To accurately describe the saturable toxic eliminations, according to kinetics of enzyme-catalyzed reactions we have proposed two toxic elimination functions - logistic and Gompertz functions in [5], in which the toxic elimination rate decreases with increasing cumulative toxic compound.

The time course of the cumulative drug toxicity $x_{3}$ with the logistic function of the drug toxic elimination is

$$
\frac{d x_{3}}{d t}=x_{2}-\eta_{L} \times x_{3}\left(\frac{1-x_{3}}{2 \theta}\right)
$$

where $\eta_{L}$ is a positive constant. The exponential [6] and logistic drug toxic elimination functions are close with a few cumulative toxic compound from drug.

The third function of the drug toxic elimination to be proposed is the Gompertz function in the time course of the cumulative drug toxicity:

$$
\frac{d x_{3}}{d t}=x_{2}-\eta_{G} \times x_{3} \ln \left(\frac{2 \theta}{x_{3}}\right),
$$

where $\eta_{G}$ is a positive constant. As time increasing the speed of the drug toxic elimination approaches a stable level and $\theta$ is called the maximal longanimous toxicity of the drug in the body (also known as the carrying capacity of the 


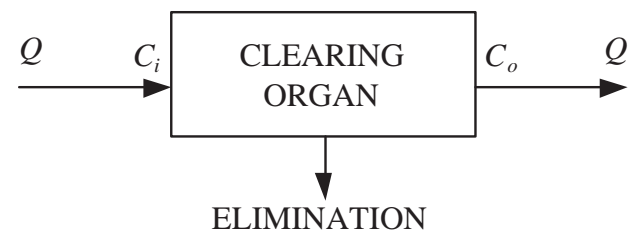

Figure 2: Physiological flow model for a perfused organ that is capable of drug toxic elimination. $Q$ denotes blood flow rate; $C_{i}$ and $C_{o}$ are concentration.

toxic compound from the drug). For human and animal treatments, the maximal longanimous toxicity of the drug is above the level that is lethal to the host. We set the constraint of the cumulative drug toxicity to $x_{3}<\theta$. As shown in Figure 1, three monotonically increasing functions are used to describe the drug toxic elimination processes. The exponential, logistic and Gompertz functions give the fit for nonsaturable and saturable toxic elimination processes respectively. We have separately combined Equations (9), (10) and (11) with Equations (1) and (2) to construct the drug scheduling models [5] [6]. The simulation solutions of these models are consistent with the clinical experience.

\section{NEW DRUG SCHEDULING MODEL WITH DIFFERENT TOXIC ELIMINATION PROCESSES}

With the above types of the drug toxic elimination models, the time course of the cumulative drug toxicity between organs and tissues is simulated on the basis of regional blood flow rates, diffusion of drug between blood and tissue, and the affinity of drug for particular organs. Their fundamental component of toxicokinetic modeling is that of organ clearance. As shown in Figure 2, this may be best illustrated by considering a single organ that is perfused by blood and is capable of eliminating the drug. $Q$ is blood flow rate through the organ and $C_{i}$ and $C_{o}$ are drug concentrations entering and leaving the organ $\left(C_{i}>C_{o}\right)$. These models with the different elimination components provide the basic units of the physiological toxicokinetic models.

Although the basic units of the physiological toxicokinetic models are relatively simple, whole-body physiological models are complex and may be represented in general form as shown Figure 3. The liver and kidney are the primary detoxification and elimination organs - they eliminate the drug toxicity from our body. Drugs passing through the liver are eliminated in a chemically altered (metabolized) form in the bile. Whenever, drug metabolism or movement across the liver involves an active process, then the likelihood of saturable kinetics exists. Thus, the logistic and Gompertz functions give the fit for saturable toxic elimination processes in the liver. On the other hand, drugs,particularly water-soluble drugs and their metabolites, are also eliminated by the kidney in urine. The kidney is a filter that cleanses toxins from our blood and its ability to excrete the toxic compounds depends on the amount of the drug toxicity in the bloodstream. Therefore, the renal excretion (i.e., filtration and passive reabsorption) may be best considered to the nonsaturable mechanisms and described by the exponential function.

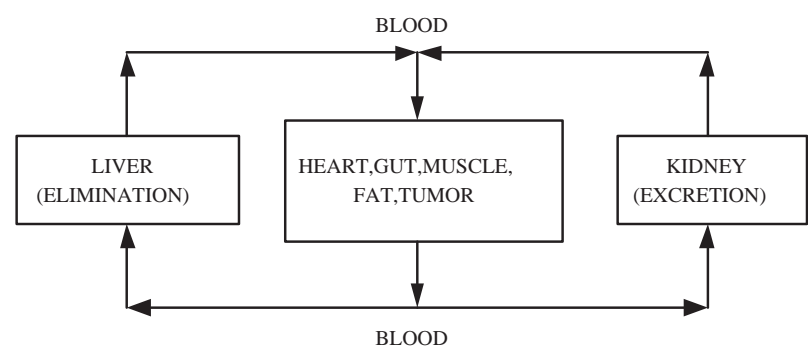

Figure 3: General physiological toxicokinetic model.

For really accurate modeling, one needs to take into consideration not only the drug toxic eliminations by single organs, but also their combination. Consequently, The time course of the cumulative drug toxicity $x_{3}$ with the drug toxic elimination in the whole-body is

$$
\frac{d x_{3}}{d t}=x_{2}-\eta_{L} \times x_{3}\left(1-\frac{x_{3}}{2 \theta}\right)-\eta_{G} \times x_{3} \ln \left(\frac{2 \theta}{x_{3}}\right)-\eta_{E} \times x_{3},
$$

where $\eta_{L}, \eta_{G}, \eta_{E}$ are nonnegative constants. We set the constraint of the cumulative drug toxicity to $x_{3}<\theta$.

We combine Equation (12) with Equations (1) and (2) to construct a new drug scheduling model. Incorporating the different toxic elimination processes into the drug scheduling model tends to make a complex situation even more so. The number of parameters increases, and the uncertainty of the model also increases. However, this type of model is used successfully. For a drug that is eliminated from the body by a single, nonsaturable metabolic pathway, an appropriate cumulative drug toxicity model can be set $\eta_{L}=\eta_{G}=0$ and $\eta_{E} \neq 0$. For a drug that is cleared by a saturable pathway and also by other nonsaturable pathways, the situation may be described by the model with $\eta_{L} \neq 0, \eta_{G} \neq 0$ and $\eta_{E} \neq 0$.

\section{EVOLUTIONARY DRUG SCHEDULING VIA THE AEGA}

In this section we use an adaptive elitist-population based genetic algorithm (AEGA), which is an efficient multimodal optimization algorithm [4], to implement the automation of the drug scheduling models for exploring multiply efficient drug scheduling policies. Therefore, a doctor expects to select a different drug scheduling policy for a different patient in the clinical treatment.

The AEGA includes two main genetic operators: the elitist crossover operator and the elitist mutation operator.

- Elitist crossover operator: If the parents and their offspring are located in the same basin of attraction, the elitist crossover operator will only select the better one of them to the next generation to reduce the population's redundancy;

- Elitist mutation operator: If an offspring is located in an unexplored basin of attraction, the elitist mutation operator will conserve the offspring and its parent together to the next generation to maintain and improve the population's diversity.

Thus, the adaptive elitist-population search technique can adaptively adjust the population size according to the features of the multimodal problem to achieve: $(i)$ A dynamic 
Table 1: The two-points crossover operator for the cycle-wise variable representation

\begin{tabular}{|c|c|c|}
\hline Parents: & $\begin{array}{l}\left\{57.05,\left.\right|_{r_{1}}\right. \\
\left\{36.98,\left.\right|_{r_{1}}\right.\end{array}$ & $\begin{array}{c}\left.(3 \times \overline{10.8}),\left.\quad\right|_{r_{2}}(81 \times \overline{10.8})\right\} \\
\left.11.56,(2 \times \overline{0}),\left.\quad\right|_{r_{2}}(40 \times \overline{0,21.39})\right\}\end{array}$ \\
\hline Offspring: & $\begin{array}{l}\left\{57.05,||_{r_{1}}\right. \\
\left\{57.05, \mid r_{1}\right. \\
\left\{57.05,||_{r_{1}}\right. \\
\left\{36.98,||_{r_{1}}\right. \\
\left\{36.98, \mid{ }_{r_{1}}\right. \\
\left\{36.98,||_{r_{1}}\right.\end{array}$ & $\begin{array}{cll}(3 \times \overline{10.8}), & \left.\mid r_{r_{2}}(40 \times \overline{0,21.39})\right\} \\
11.56,(2 \times \overline{0}), & \left.\left.\right|_{r_{2}}(81 \times \overline{10.8})\right\} \\
11.56,(2 \times \overline{0}), & \left.\left.\right|_{r_{2}}(40 \times \overline{0,21.39})\right\} \\
11.56,(2 \times \overline{0}), & \left.\mid r_{2}(81 \times \overline{10.8})\right\} \\
(3 \times \overline{10.8}), & \left.\left.\mid r_{2}(81 \times \overline{10.8})\right\}\right\} \\
(3 \times \overline{10.8}), & \left.\mid r_{2}(40 \times \overline{0,21.39})\right\}\end{array}$ \\
\hline
\end{tabular}

number of individuals in the population so that only a minimum number of elitist individuals is used to search for each optimum; and (ii) All the individuals in the population searching for different optima in parallel.

The AEGA has been experimentally tested with a difficult test suite in [4] [6], and all experiments have demonstrated that the AEGA consistently and significantly outperforms the other multimodal evolutionary algorithms in efficiency and solution quality.

\subsection{A Cycle-wise Variable Representation}

Here, we use a cycle-wise variable representation to accurately and efficiently describe the drug scheduling policy in a chromosome.

Definition 1: Due to the large number of variables and fine accuracy involved, a cycle-wise variable representation of the drug scheduling is defined by

$$
\begin{array}{cl}
\text { Variable representation } & :=\left\{[C \mid D]^{*} \mid[D(D C)]^{*}\right\} \\
C & :=\left[c_{i}\right]^{*} \\
D & :=k_{i} \times \overline{d_{i}, \cdots, d_{j}}
\end{array}
$$

where $c_{i}, d_{i}, d_{j}$ are the drug doses on each day, $k_{i}$ is the number of cycles, $\overline{d_{i}, \cdots, d_{j}}$ is the repetend and "*" represents the repetition of the structure that is located in the front square bracket, but the values can be different.

By Definition 1, the cycle-wise variable representation includes two parts: a front and a cyclic parts. The front part is $[C \mid D]^{*}$. It describes the drug doses in the initial treatment days. The cyclic part is $[D(D C)]^{*}$. It consists of the number of cycles $k_{i}$ and the repetend $\left(\overline{d_{i}, \cdots, d_{j}}\right)$. For example, the cycle-wise variable representation is $\{57.05$, $27 \times \overline{(21.5,2 \times \overline{0})}\}$ that means giving the drug $57.05 \mathrm{D}$ at the first day and then repeated 27 times giving the drug $21.5 \mathrm{D}$ every three days. The cycle-wise variable representation is very suitable for the drug scheduling problem. Because in the first few days of the treatment period, the patient's body may not have adapted to the drug, but it is important to kill as many tumor cells as possible, the drug doses will be adjusted day by day. We use the front part to represent the drug doses in this initial period. Then when the patient's body gradually gets used to the drug, the drug administration schedule will follow a fixed cycle and a fixed dose pattern, which is suitably represented by the cycle-part.

\subsection{Elitist Crossover Operator}

Here we combine the standard multi-points (two-points) crossover operator with the adaptive elitist-population search techniques to construct the elitist crossover operator for the cycle-wise variable representation. Let $r_{1}$ and $r_{2}$ be the crossover points in the front and the cyclic parts respectively of the two parents selected randomly from the population. The offspring are produced by taking all the combinations of the 3 segments (separated by $r_{1}, r_{2}$ ) of the parents' representations. In Table 1, the two-points crossover operation generally can generate 6 offspring to improve the successful rate in the search process. Then two better solutions, which satisfy all the constraints, are selected from the parents and their offspring to the next generation.

Before we carrying out the crossover operation, the adaptive elitist-population search technique which was incorporated into the crossover operator will delete the worse one from the two selected parents to reduce the population's redundancy, if they are located in the same basin of attraction. Of course, if this is carried out, no standard crossover operation will be performed.

\subsection{Elitist Mutation Operator}

In an elitist mutation operator, the basic mutation works as follows: for a randomly chosen position in the cycle-wise representation, replaces its value with another randomly chosen value (not the same as the one to be replaced) with certain mutation probability. For example, in Table 2, the third point of the parent is changed from 7.6 to 10.8 to generate its offspring (1); or the last value of the last cyclic part of the parent is changed from 19.58 to 21.64 to generate its offspring (2); or the number of cycles in the inner cycle of the last cyclic part is changed from 2 to 3 to generate its offspring (3).

The elitist mutation in the AEGA is that when the offspring is located in an unexplored optimal attraction, it and its parent are conserved together to the next generation to increase the population's diversity [4], otherwise, only the best one of the parent and the offspring will be selected to the next generation.

\subsection{The AEGA for the Drug Scheduling Model}

In order to successfully explore multiple optimal solutions of the drug scheduling models, the AEGA is implemented as follows:

1. Set $t=0$ and initialize a chromosome population $P(t)$ (uniform random initialization within the bounds);

2. Evaluate $P(t)$ by using the fitness measure; 
Table 2: The one-point mutation operator for the cycle-wise variable representation

\begin{aligned} & \hline Parent: $\{57.05,3 \times \overline{7.6},(40 \times \overline{0,19.58})\} \\ &$\hline Offspring: \\ &$(1):\{57.05,(7.6,10.8,7.6),(40 \times \overline{0,19.58})\} \\ &$ or \\ &$(2):\{57.05,(3 \times \overline{7.6}),(40 \times \overline{(0, \overline{21.64})}) \\ &$ or \\ &$(3):\{57.05,(2 \times \overline{7.6}),(27 \times \overline{0,0,19.58})\} \\ &$\hline\end{aligned}

3. While (termination condition not satisfied) Do

(a) Elitist crossover operation to generate $P(t+1)$;

(b) Elitist mutation operation to generate $P(t+1)$;

4. Evaluate $P(t+1)$;

5. Stop if the termination condition is satisfied; otherwise, $t \longleftarrow t+1$ and go to Step 3 ;

The drug scheduling problems were simulated using the AEGA with the following parameters: initial population size $=2000$; generation size $=20000$; crossover rate $=1.0$; mutation rate $=1.0$ and the distance threshold $\sigma=10$. The drug scheduling model was simulated using numerical differentiation method of Runge-Kutta [12], with a small time interval of 0.01 day for good accuracy. In the new model, we add three parameters $\eta_{L}, \eta_{G}, \eta_{E}$ and $\theta$. Here we set these parameters as $\eta_{L}=\eta_{G}=\eta_{E}=0.2$ and $\theta=100$.

\section{SIMULATION RESULTS UNDER THE NEW MODEL}

Automating the new drug scheduling model via the multimodal optimization algorithm, the AEGA can obtain seven most efficient drug scheduling policies, which are listed in Table 3. The global optimal solution is illustrated in Figure 4 .

The multiple efficient drug scheduling policies under the new model match well with the clinical experience. In the clinical treatment, generally the drug scheduling policies include two categories of patterns: continuous and repeated. The optimal drug scheduling policies under the new model belong to these two categories respectively. In some patients, the aim of treatment may be to reduce the tumor size with minimum toxicity. The drug scheduling policies with the long repetend (e.g., giving drug every three days) are suitable because their cumulative drug toxicity often decreases to the low level (e.g., the third efficient drug scheduling policy obtained by the new model as shown in Figure 5 and its cumulative drug toxicity often decreases to $70 \%$ of the maximal longanimous toxicity of $\operatorname{drug} \theta$ ). For other patients, they may be cure despite higher toxicity, the continuous or repeated patterns with the short repetend drug scheduling policies (e.g., giving drug every day) are suitable because these policies are more efficient but with high toxicity (Figure 4). So these multiple efficient drug scheduling policies obtained by the new model are more useful. According to the different conditions of the patients, the doctor can select the suitable drug scheduling policy to treat cancer and get the best efficient results.

\section{PARAMETER ANALYSIS AND DISCUSSION OF THE NEW MODEL}

\subsection{Parameter Analysis of the New Model}

In the new cancer drug scheduling model, we add three drug toxic elimination rates $\eta_{L}, \eta_{G}$ and $\eta_{E}$ into the third equation and adjust the constraint threshold of the cumulative drug toxicity $x_{3}<\theta$. These parameters $\eta_{L}, \eta_{G}, \eta_{E}$ and $\theta$ are in positive values. In the above sections, we set $\eta_{L}=\eta_{G}=\eta_{E}=0.2$ and $x_{3}<100$ to study the optimal drug scheduling policies for the new model.

The parameters $\eta_{L}, \eta_{G}$ and $\eta_{E}$ describes the capability of the drug toxic elimination in the body. For some drugs, if their toxic elimination rates are high, this means that the cumulative toxicity of these drugs is easily eliminated from the body, so the best efficient drug policy should be the continuous pattern giving drug policy or repeated pattern giving drug policy with short repetend (e.g., giving drug every two days). On the contrary, if their toxic elimination rates are low, this means that the cumulative toxicity of these drugs is difficult to be eliminated from the body, so the best efficient drug policy should be the repeated pattern giving drug policy with long repetend (e.g., giving drug every seven days), because the clearance of drug toxicity is slow. This principle can be simulated by our new model. At the same time, the number of the local optimal solutions varies with different parameter settings.

As shown in the upper part of Table 4, when we fix the constraint threshold of the cumulative drug toxicity $x_{3}<$ 100 and only consider that only one parameter of $\eta_{L}, \eta_{G}$ and $\eta_{E}$ is unequal to 0 , if $\eta_{L}, \eta_{G}$ and $\eta_{E}$ are larger than 1, 0.7 and 1 respectively, the new model only has one optimal solution (continuous drug policy). This means that the clearance capability of the drug toxicity is strong in the body and can maintain the cumulative drug toxicity lower than its constraint. The continuous drug scheduling policy can be selected for the best treatment effect. If $\eta_{L}, \eta_{G}$ and $\eta_{E}$ are decreased, the model via the multimodal optimization algorithm AEGA can obtain the multiple optimal solutions. For example, if $\eta_{L}$ is decreased to 0.7 , the number of the optimal solutions increases to 4 and the repeated drug policy (giving drug every two days) is the global optimum; if $\eta_{L}$ is decreased to 0.5 , the model has 8 optimal solutions and the global optimum is to give drug every three days. if $\eta_{L}$ is decreased to 0.2 , the model has 15 optimal solutions and the global optimum is to give drug every eight days. In this case, the continuous drug policy is not an optimum due to its high cumulative toxicity. However, if $\eta_{L}, \eta_{G}$ and $\eta_{E}$ are 
Table 3: The most efficient drug scheduling policies obtained by the new model.

\begin{tabular}{clcc}
\hline No & The most efficient drug scheduling policies & $\operatorname{Index}\left(x_{1}\right)$ & No. of cells $\left(\times 10^{4}\right)$ \\
\hline$(1)$ & $\{25.04,10.30,0,(81 \times \overline{8.14})\}$ & 17.62 & 2.23 \\
$(2)$ & $\{26.22,20.39,41 \times \overline{(0,16.16)}\}$ & 17.56 & 2.36 \\
$(3)$ & $\{57.05,0,10.14,0,27 \times \overline{(23.98,(2 \times \overline{0})})\}$ & 17.39 & 2.80 \\
$(4)$ & $\{32.92,(3 \times \overline{0}), 20 \times \overline{(31.46,(3 \times \overline{0})})\}$ & 17.09 & 3.78 \\
$(5)$ & $\{46.88,(3 \times \overline{0}), 16 \times \overline{(38.51,(4 \times \overline{0})})\}$ & 16.72 & 5.48 \\
$(6)$ & $\{46.07,(5 \times \overline{0}), 13 \times \overline{(45.04,(5 \times \overline{0})})\}$ & 16.12 & 9.98 \\
$(7)$ & $\{57.05,(6 \times \overline{0}), 11 \times \overline{(48.43,(6 \times \overline{0})})\}$ & 14.94 & 32.5 \\
\hline
\end{tabular}

(a)

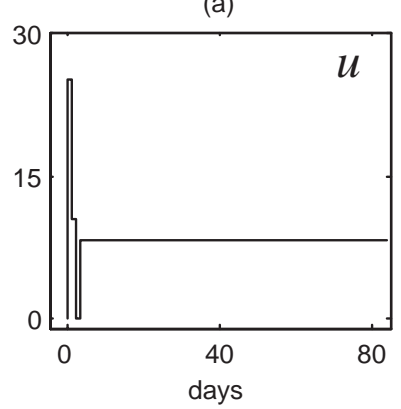

(b)

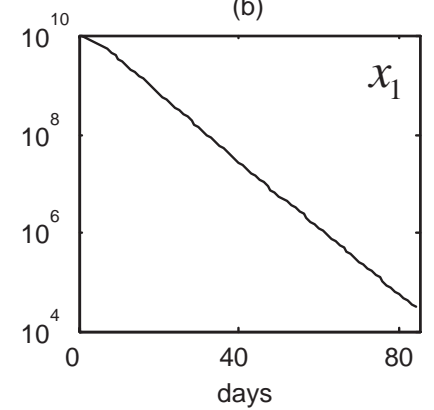

(c)

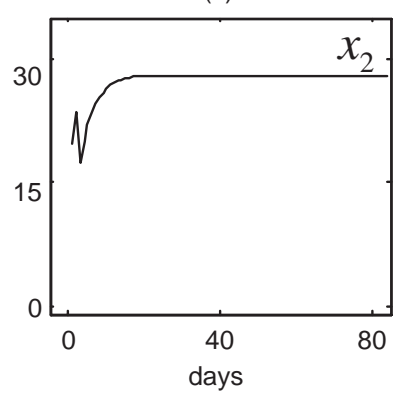

(d)

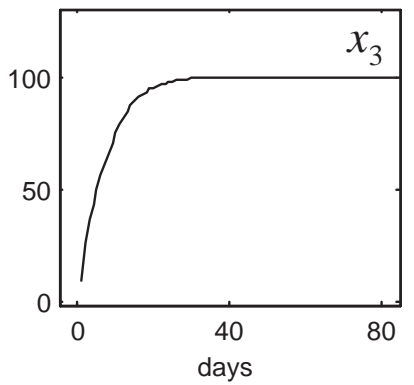

Figure 4: The best drug scheduling policy obtained by the new model. The sub-figures (a), (b), (c) and (d) show the control variable $u$, the final mass of the tumor (inversely related to the best performance index $x_{1}$ ), the change patterns of the drug concentration $x_{2}$ and the cumulative drug toxicity $x_{3}$ in the 84 days respectively.

(a)

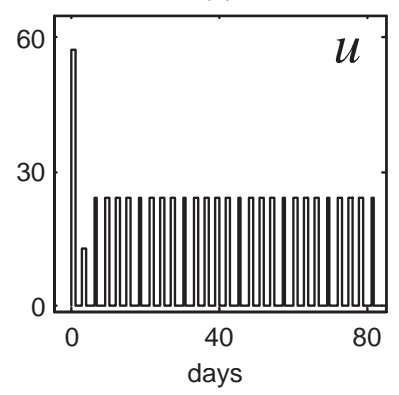

(b)

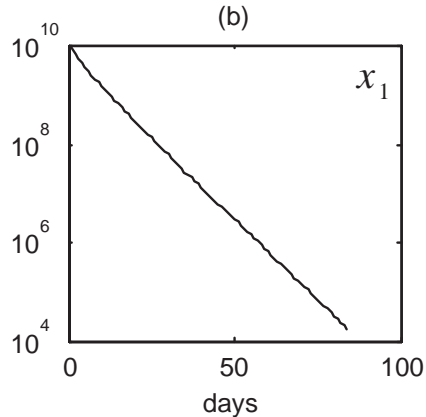

(c)

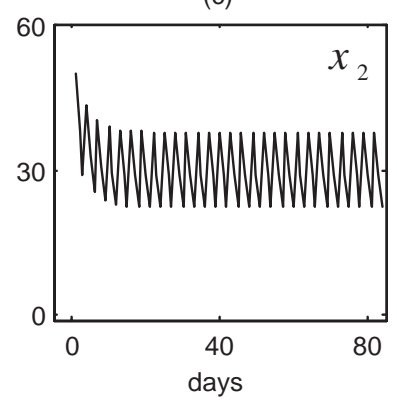

(d)

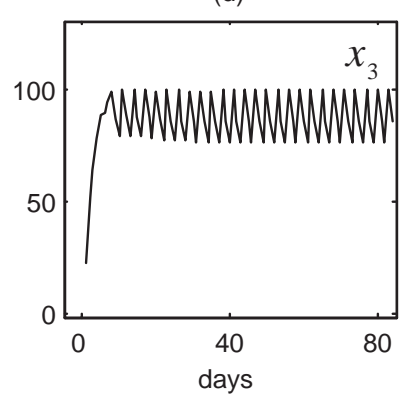

Figure 5: The third optimal drug scheduling policy obtained by the new model. The captions of sub-figures (a), (b), (c) and (d) are same as Fig. 4. 
decreased to $0.1,0.1$ and 0.2 respectively, the model has no efficient solution, because at this time the drug toxicity is too difficultly to be eliminated from the body.

For the cases that all parameters $\eta_{L}, \eta_{G}$ and $\eta_{E}$ are unequal to 0 , we can get similar results. If $\eta_{L}, \eta_{G}$ and $\eta_{E}$ are equal to 0.4 , the new model only has one optimal solution (continuous drug policy). If $\eta_{L}, \eta_{G}$ and $\eta_{E}$ are decreased to 0.2 , the number of the optimal solutions increases to 7 , and if $\eta_{L}, \eta_{G}$ and $\eta_{E}$ are 0.1 , the model has no efficient solution.

Table 4: The most efficient drug scheduling policies obtained by the new model with different parameters settings

\begin{tabular}{|c|c|c|c|c|}
\hline$\eta_{L}$ & $\eta_{G}$ & $\eta_{E}$ & $\theta$ & $\begin{array}{c}\text { Number of } \\
\text { optimal solutions }\end{array}$ \\
\hline 1 & 0 & 0 & 100 & 1 \\
\hline 0.9 & 0 & 0 & 100 & 2 \\
\hline 0.7 & 0 & 0 & 100 & 4 \\
\hline 0.5 & 0 & 0 & 100 & 8 \\
\hline 0.2 & 0 & 0 & 100 & 15 \\
\hline 0.1 & 0 & 0 & 100 & None \\
\hline 0 & 0.7 & 0 & 100 & 1 \\
\hline 0 & 0.6 & 0 & 100 & 2 \\
\hline 0 & 0.4 & 0 & 100 & 5 \\
\hline 0 & 0.3 & 0 & 100 & 9 \\
\hline 0 & 0.1 & 0 & 100 & None \\
\hline 0 & 0 & 1 & 100 & 1 \\
\hline 0 & 0 & 0.4 & 100 & 4 \\
\hline 0 & 0 & 0.2 & 100 & None \\
\hline 0.4 & 0.4 & 0.4 & 100 & 1 \\
\hline 0.4 & 0.1 & 0.2 & 100 & 7 \\
\hline 0.4 & 0.2 & 0.1 & 100 & 10 \\
\hline 0.4 & 0.1 & 0.1 & 100 & 12 \\
\hline 0.2 & 0.1 & 0.4 & 100 & 3 \\
\hline 0.2 & 0.4 & 0.2 & 100 & 6 \\
\hline 0.2 & 0.2 & 0.2 & 100 & 7 \\
\hline 0.2 & 0.2 & 0.1 & 100 & 9 \\
\hline 0.1 & 0.2 & 0.4 & 100 & 2 \\
\hline 0.1 & 0.1 & 0.4 & 100 & 3 \\
\hline 0.1 & 0.4 & 0.2 & 100 & 6 \\
\hline 0.1 & 0.1 & 0.1 & 100 & None \\
\hline 0.2 & 0.2 & 0.2 & 200 & 1 \\
\hline 0.2 & 0.2 & 0.2 & 150 & 4 \\
\hline 0.2 & 0.2 & 0.2 & 120 & 5 \\
\hline 0.2 & 0.2 & 0.2 & 100 & 7 \\
\hline 0.2 & 0.2 & 0.2 & 80 & 13 \\
\hline 0.2 & 0.2 & 0.2 & 40 & None \\
\hline
\end{tabular}

On the other hand, when we fix the drug toxic elimination rates $\eta_{L}, \eta_{G}, \eta_{E}$ and change the constraint thresholds of the cumulative drug toxicity $x_{3}<\theta$, where $\theta$ is the maximal longanimous toxicity of drug. For some drugs, if their $\theta$ are large, which indicates that if the toxicity of these drugs is not too strong in the body, the best efficient drug policy should be the continuous drug policy or repeated drug policy with short repetend; On the contrary, if their $\theta$ is small, which indicates that the cumulative toxicity of these drugs is strong to the body, so the best efficient drug policy should be the repeated drug policy with long repetend to eliminate the drug toxicity by the clearance. This principle can also be simulated by our new model with the logistic toxicity clear- ance. Moreover, the number of the local optimal solutions varies as the parameters changes.

As shown in the second part of Table 4, when we set the constraint threshold of the cumulative drug toxicity $\eta_{L}=$ $\eta_{G}=\eta_{E}=0.2$, if $\theta=200$, the model has only one optimal solution (continuous drug policy). For this case, the drug toxicity is low and the constraint of the cumulative drug toxicity is loosened, the continuous policy is the most efficient drug scheduling policy in the clinical treatment. If $\theta$ is decreased to 150 , the model has 4 optimal solutions and the global optimum is to give drug every two days. If $\theta$ is decreased to 120 , the model has 5 optimal solutions and the global optimum is the repeated drug policy (giving drug every two days). If $\theta$ is decreased to 80 , the model has 13 optimal solutions and the global optimum is to give drug every three days. If $\theta$ is decreased to 40 , the model has no efficient solution. This means the drug concentration $x_{2}$ of the optimal drug policy which satisfies $x_{3}<40$, is always smaller than the threshold $\beta=10$, below which the drug is not efficient. So the tumor cells increase in the treatment period and there is, in fact, no acceptable solution for this case. Therefore, the drug with the constraint $x_{3}<40$ cannot be used effectively to treat cancer, because its toxicity will be exceeded.

\subsection{Discussion on the New Model}

Cancer cells can be divided into proliferating or cycling cells and nonproliferating or quiescent cells. The entire process of cell division (the cell cycle) begins following mitosis and is conventionally divided into four phases: mitosis $(M)$, first gap $\left(G_{1}\right)$, DNA synthetic phase $(S)$ and second gap $\left(G_{2}\right)$. According to the effect on the cell cycle, anticancer drugs can be classified into non-phase-specific and phase-specific drugs. For example, Alkylating agents, Nitrosoureas, Anthracyclines, Dacarbazine (DTIC), Mitomycin C, Actinomycin D, Cisplatinum and Carboplatinum are non-phase-specific anticancer drugs. They have been shown to have activity against cells at all phases of the cell cycle. Vinca alkaloids, hydroxyurea, Cytosine arabinoside, Methotrexate, 6-Mercaptopurine, 6-Thioguanine, Procarbazine, VM-26 and VP16-213 are phase-specific anticancer drugs. They act on a specific phase of cycling cells.

For non-phase-specific anticancer drugs, our new models can be directly used to simulate the drug administration process because these models concern the cancer cells as a whole population. For phase-specific anticancer drugs, other kinds of cancer chemotherapy models [9] [11] [14] can be used as they divide the tumor cells into different phasesubpopulations. However, the proportions of different phasesubpopulations in the tumor are difficult to evaluate in the clinical treatment.

In our current study, the data used to solve the problems numerically are mainly synthetic. This means that the data and the parameters were artificially chosen to represent generic and typical treatments of a solid tumor by an anticancer drug, as opposed to data based upon clinical measurements.

Our last author, who is an oncologist, has completed a randomized phase II study comparing the combination of gemcitabine and cisplatin with gemcitabine and etoposide. All data related to drug administration, dosage, tumor response and toxicity are prospectively collected. In our future work, we will select data from real clinical trials and adjust 
the equations, coefficients and constraints of our new models to simulate the pharmacokinetics, pharmacodynamics and toxicity of the anticancer drugs accordingly.

\section{CONCLUSION AND FUTURE WORK}

In this paper, new optimal control models of drug scheduling in cancer chemotherapy were proposed and an adaptive elitist-population based genetic algorithm (AEGA) was applied to solve above models. Through incorporating a toxicity clearance term into Martin's drug scheduling model, the previous modified model takes into account the body's ability of recovering from the effect of the drug and match well with the clinical treatment experience. As different drugs have different toxicity clearance processes, in this paper, we further proposed two new drug scheduling models with different toxicity clearance according to the kinetics of the enzyme-catalyzed chemical reactions. They are logistic and Gompertz drug toxicity clearance functions, which give the fit for the phenomenon of saturation with substrate in enzymatic reactions. For exploring multiple efficient drug scheduling policies, we use a multimodal algorithm AEGA to solve the new models, and discuss the situation of multiple optimal solutions under different parameter settings. The simulation results obtained by the new models match well with the clinical treatment experience, and can provide much more drug scheduling policies for the doctor to choose depending on the individual conditions of the patients.

In our future work, first we will use data from real clinical trials on single drugs, adjust the coefficients of our new models and simulate the pharmacokinetics, pharmacodynamics and toxicity of the drug. Then we will focus on the modeling multi-drug chemotherapy, which aims to predict drug combinations, doses, and schedules to effectively reduce tumor cells and prolong patient life. Computational models may become important tools to help optimize and predict cancer treatments.

\section{ACKNOWLEDGMENTS}

This research was partially supported by RGC Earmarked Grant 4173/04E of Hong Kong SAR and RGC Research Grant Direct Allocation of the Chinese University of Hong Kong.

\section{REFERENCES}

[1] B. Bojkov, R. Hansel, and R. Luus, Application of direct search optimization to optimal control problem, Hung. J. Ind. Chem. 21: 177-185, 1993
[2] E. F. Carrasco, and J. R. Banga, A hybrid method for the optimal control of chemical processes, Proc. of UKACC Int. Conf. Control'98, II: 925-930, June 1998

[3] E. M. David, Biochemistry, Harcourt/Academic Press, 2001

[4] K. S. Leung, and Y. Liang, Genetic algorithm with adaptive elitist-population strategies for multimodal function optimization. Proc. of Int. Conf. GECCO-2003, pages 1160-1171. July 2003

[5] K. S. Leung, Y. Liang, and S. K. Mok, Multiple optimal scheduling in cancer chemotherapy by evolutionary computation. Proc. of Taiwan-Japan Symposium 2005 on Intelligent Technology and Innovational Computing, pages 9-17, August 2005

[6] Y. Liang, K. S. Leung, and S. K. Mok, Evolutionary Drug Scheduling Model for Cancer Chemotherapy, IEEE Trans. on Inf. Tech. in BioMed., 10(2): 237-245, 2006

[7] R. Luus, Comments on dynamic optimization of batch reactors using adaptive stochastic algorithms, Ind. Eng. Chen. Res., 37: 305-327, 1998

[8] R. B. Martin, Optimal control drug scheduling of cancer chemotherapy, Automatica, 28: 1113-1123, 1992

[9] V. Minay, and O. Gabriela, Heuristic design of cancer chemotherapies, IEEE Trans. on Evol. Comp., 8(6): 513-521, 2005

[10] J. M. Murray, Optimal control of a cancer chemotherapy problem with general growth and loss functions, Math. Biosci., 38: 273-287, 1990

[11] J. C. Panetta and J. Adam, A mathematical model of cycle-specific chemotherapy, Math. Comput. Modeling, 22(2): 67-82, 1995

[12] W. H. Press, S. A. Teukolsky, W. T. Vetterling, and B. P. Flannery, Numerical Recipes in C: he Art of Scientific Computing (2nd edition) Cambridge. Cambridge University Press, 1992

[13] K. C. Tan, E. F. Khor, J. Cai, C. M. Heng, and T. H. Lee, Automating the drug scheduling of cancer chemotherapy via evolutionary computation, Art. Intel. in Med., 25: 169-185, 2002

[14] S. M. Tse, Y. Liang, K. S. Leung, K. H. Lee, and S. K. Mok, Multiple drugs cancer chemotherapy scheduling by a new memetic optimization algorithm, Proceeding of 2005 IEEE Congress on Evol. Comp. pages 699-706, August 2005 